\title{
Loop Variables in Topological Gravity
}

\author{
by \\ Y. Bi and J. Gegenberg \\ Department of Mathematics and Statistics \\ University of New Brunswick \\ Fredericton, New Brunswick \\ CANADA E3B 5 A3
}

\begin{abstract}
We examine the relationship between covariant and canonical (Ashtekar/Rovelli/Smolin) loop variables in the context of BF type topological field theories in $2+1$ and $3+1$ dimensions, with respective gauge groups $\mathrm{SO}(2,1)$ and $\mathrm{SO}(3,1)$. The latter model can be considered as the simplest topological gravity theory in $3+1$ dimensions. We carry out the canonical quantization of this model in both the connection and loop representations, for the two spatial topologies $T^{3}$ and $S^{2} \times S^{1}$.
\end{abstract}

March 1993

UNB Technical Report 93-02 


\section{Introduction}

An important advance in the quest to construct a viable and realistic quantum theory of gravity was made by Ashtekar and his coworkers when they recast and slightly generalized Einstein gravity by introducing new canonical variables [1, 2]. In these new variables, the constraints are polynomial and can be solved explicitely in terms of non-local, but gauge invariant, "loop variables" $T^{n}$ [2, 3]. These loop variables are not the physical observables in 4 -D gravity since they are not diffeomorphism invariant [幽. The discovery of non-trivial physical observables is an outstanding problem in the non-perturbative quantization of 4-D Einstein gravity.

In three dimensional gravity theory the problem of constructing physical observables is at least partially solved. The reason for this is, essentially, that three dimensional gravity is a topological field theory [5]. Indeed, if we relax the non-degeneracy of the spacetime triad, in the case of zero cosmological constant, 3-D Einstein gravity (in the first order formalism) is equivalent to Chern-Simons theory with gauge group $I S O(2,1)$. In fact, Einstein gravity in the first order formalism is the prototype of another interesting topological field theory- the BF theories [6, 7]. With non-zero cosmological constant, Einstein gravity is equivalent to a Chern-Simons theory with gauge groups $\mathrm{SO}(3,1)$ and $\mathrm{SO}(2,2)$, respectively, in the cases of positive and negative cosmological constant. The equations of motion (or constraints) imply that the $\operatorname{ISO}(2,1)$ connection in the Chern-Simons model is flat, i.e., locally pure gauge. One may now construct gauge invariant observables that do not vanish when the constraints are imposed and which are diffeomorphism invariant (due to the fact that the diffeomorphisms are generated by the $\operatorname{ISO}(2,1)$ gauge transformations in this case [5]). These are physical observables, denoted generically here by $W$, the "covariant loop variables" of the system. The canonical quantization of 3-D Einstein gravity in first order formalism can be handled very much analogously to the $4-\mathrm{D}$ case [8]. In particular, the constraints can be solved in terms of the 3-D analogues of the $T^{n}$, i.e., "Ashtekar" loop variables.

In the next section we will elucidate the relationship between the two species of global observables encountered in 3-D gravity theory: 
the covariant loop variables and the Ashtekar loop variables. In section 3. we will review the properties of $4-\mathrm{D}$ BF theory with gauge group $\mathrm{SO}(3,1)$. This theory may be viewed as the closest topological field theory relative of 4-D Einstein gravity [6, 9] . We will canonically quantize this theory in terms of the Ashtekar loop variables $T^{n}$ and relate the latter to the covariant loop variables of the theory. Finally, in section 4 . we will first of all use the results obtained to explicitely carry out the quantization of this model for the special cases where 3-space has the topologies $T^{3}$ and $S^{2} \times S^{1}$, and second we will discuss the implications for non-topolocical quantum gravity in 4-D.

\section{3-D Gravity}

We begin by reviewing 3-D Einstein gravity in the form considered by Witten [5]. In (2+1)-dimensions, the Einstein-Hilbert action can be written in the form [5]

$$
S=\frac{1}{2} \int d^{3} x \epsilon^{i j k} \epsilon_{a b c} e_{i}^{a} R_{j k}^{b c}
$$

where lower case latin indices from the beginning of the alphabet are $S O(2,1)$ indices, while those from the middle are spacetime indices. The $e_{i}^{a}$ are an orthonormal triad and $\omega_{i}^{a b}$ are the spin connection components with respect to the group $S O(2,1)$. The curvature is given by

$$
R_{i j}^{a b}=\partial_{i} \omega_{j}^{a b}-\partial_{j} \omega_{i}^{a b}+\left[\omega_{i}, \omega_{j}\right]^{a b},
$$

and the classical equations of motion are

$$
\begin{array}{r}
D_{i} e_{j}^{a}-D_{j} e_{i}^{a}=0, \\
R_{i j}^{a b}=0 .
\end{array}
$$

Here $D_{i}$ is the covariant exterior derivative with respect to the connection $\omega$. The equations of motion imply that the spacetime is a flat Lorentzian manifold. The gauge group of the system is the Poincaré group $I S O(2,1)$. Let $J^{a b}$ be Lorentz generators and $P^{a}$ the translations, so that the gauge potential for the group $\operatorname{ISO}(2,1)$ is the one-form

$$
A_{i}=e_{i}^{a} P_{a}+\omega_{i}^{a} J_{a}
$$


Here we have defined $\omega_{i}^{a}:=1 / 2 \epsilon^{a b c} \omega_{i b c}$ and $J_{a}:=1 / 2 \epsilon_{a b c} J^{b c}$. The generators $P_{a}, J_{a}$ determine a quadratic form on the Lie algebra

$$
<J_{a}, J_{b}>=<P_{a}, P_{b}>=0, \quad<J_{a}, P^{b}>=\delta_{a}^{b} .
$$

The generators of $I S O(2,1), P_{a}$ and $J_{a}$, satisfy the algebra

$$
\begin{array}{r}
{\left[J_{a}, P_{b}\right]=\epsilon_{a b c} P^{c},} \\
{\left[J_{a}, J_{b}\right]=\epsilon_{a b c} J^{c},} \\
{\left[P_{a}, P_{b}\right]=0 .}
\end{array}
$$

The Chern-Simons functional for the $\operatorname{ISO}(2,1)$ gauge connection $A_{i}$,

$$
I_{C S}=\int_{M} \operatorname{Tr}\left(A \wedge d A+\frac{2}{3} A \wedge A \wedge A\right),
$$

precisely coincides with the Einstein-Hilbert action (11), as follows from equations (5)-(7). Hence (2+1)-dimensional gravity is a ChernSimons gauge field theory.

We perform a canonical analysis in order to display the constraints. Let $M=R \times \Sigma$, where $\Sigma$ is a closed 2-manifold. The action can be written as

$$
\begin{array}{r}
S=\int d t \int_{\Sigma} d^{2} x\left(\epsilon^{a b c} \dot{\omega}_{b c}^{\mu} \epsilon_{\mu \nu} e_{a}^{\nu}\right. \\
\left.+\frac{1}{2} \epsilon^{a b c} \omega_{0 b c} \epsilon^{\mu \nu} D_{\mu} e_{\nu a}+\frac{1}{2} \epsilon^{a b c} \epsilon^{\mu \nu} e_{0 a} R_{\mu \nu}^{b c}\right),
\end{array}
$$

where the $\epsilon^{\mu \nu}:=\epsilon^{0 \mu \nu}$, and the dot "." denotes differentiation with respect to $x^{0}$. The 0 -components of the fields are Lagrange multiplers, which impose the constraints

$$
\begin{aligned}
& D_{[\mu} e_{\nu]}^{a} \approx 0, \\
& R_{\mu \nu}^{a b} \approx 0,
\end{aligned}
$$

where $\mu, \nu=1,2$ are spatial indices. Note that they are of the same form as the equations of motion of the gauge fields $(e, \omega)$. From the action (11), the fundamental Poisson bracket can be read off as

$$
\left\{\omega_{\mu}^{a}(x), \tilde{e}_{b}^{\nu}(y)\right\}=\delta_{b}^{a} \delta_{\mu}^{\nu} \delta^{2}(x, y),
$$


where $\tilde{e}_{b}^{\nu}=\epsilon^{\nu \rho} e_{\rho b}$ is a density.

In $2+1$ gravity, the covariant gauge invariant observables are the Wilson loop variables of the connection $A_{i}=e_{i}^{a} P_{a}+\omega_{i}^{a} J_{a}$ :

$$
W_{R}(C)=\operatorname{Tr}_{R} P \exp \left\{\oint_{C}\left(e_{i}^{a} P_{a}+\omega_{i}^{a} J_{a}\right) d x^{i}\right\},
$$

i.e., the $\operatorname{ISO}(2,1)$ holonomy operators [5]. The spacetime curve $C$ is closed and $R$ is a representation of $I S O(2,1)$.

Variables analogous to those introduced by Ashtekar in the $3+1$ dimensional case [1] can be constructed in 2+1-D theory [8]. From the Hamiltonian form of the Einstein-Hilbert action, a class of loop variables on the conjugate variables $(e, \omega)$ can be defined. The first of these observables is the spatial Wilson loop of $\omega$ ( the $S O(2,1)$ connection):

$$
T_{r}^{0}[C]:=\operatorname{Tr}_{r} P \exp \left[\oint_{C} \omega\right]=\operatorname{Tr}_{r} P \exp \left[\oint_{C} d s^{\mu} \omega_{\mu}^{a} J_{a}\right],
$$

where $r$ denotes a representation of $S O(2,1)$ and $C$ is a loop in space. The remaining variables, denoted $T_{r}^{n}[C]$, depend on the $e$-variables. They are obtained by inserting $n e$-variables along the holonomy of a loop:

$$
\begin{array}{r}
T_{r}^{n}[C]=\int d s^{\mu_{1}} \cdots \int d s^{\mu_{n}} \sum_{p} \theta\left(s_{q}-s_{p}\right) \cdots \theta\left(s_{j}-s_{i}\right) \times \\
\operatorname{Tr}_{r}\left[e_{\mu_{i}}\left(C\left(s_{i}\right)\right) U_{C}\left(s_{i}, s_{j}\right) e_{\mu_{j}}\left(C\left(s_{j}\right)\right) \cdots e_{\mu_{p}}\left(C\left(s_{p}\right)\right) U_{C}\left(s_{p}, s_{i}\right)\right],
\end{array}
$$

where the summation is over the permutation of $n$ indices and $\theta\left(s_{i}-\right.$ $\left.s_{j}\right)$ are step functions. In the following, we omit the representation subscript $r$. The quantity $U_{C}$ is defined as

$$
U_{C}(s, t):=P \exp \left[\int_{s}^{t} d s^{\mu} \omega_{\mu}^{a} J_{a}\right]
$$

The $T^{n}$ 's form a closed graded Poisson algebra, which has the structure

$$
\left\{T^{n}, T^{m}\right\} \sim T^{n+m-1} .
$$

From this we can see that $T^{0}$ and $T^{1}$ form a subalgebra. In the case of three dimensional gravity, we need only consider the $T^{0}, T^{1}$ variables. 
From Ref. 80 we know that the Poisson brackets of the observables with the constraints are weakly zero. Hence $T^{0}, T^{1}$ are physical observables for pure $2+1$ gravity. When gravity is coupled to ordinary local matter, then $T^{0}, T^{1}$ are no longer the physical observables because they fail to commute with all the constraints. However they are still gauge invariant and very important quantities for the construction of the loop representation [1, 10].

Now we have two kinds of observables-Ashtekar $T$ variables and the covariant loop variables $W$ - for $2+1$ gravity theory. We now discuss the relation between these two kinds of observables in the canonical formulation of the theory. The loops $C$ are taken to be at fixed time, i.e. they lie in a two-dimensional spatial hypersurface $\Sigma_{t}$. So in the covariant loop variables

$$
W(C)=\operatorname{Tr} P \exp \left\{\oint_{C}\left(e_{\mu}^{a} P_{a}+\omega_{\mu}^{a} J_{a}\right) d x^{\mu}\right\}
$$

the curves $C$ are spatial, as are the loops in the Ashtekar $T$ variables. Since the $I S O(2,1)$ group manifold is the total space of the cotangent bundle of the $S O(2,1)$ group manifold [5], we can express the translation generators $P_{\mu}$ in terms of the rotation generators $J_{\mu}$ and an infinitesimal parameter $\theta$ [11:

$$
P_{\mu}=\theta J_{\mu}
$$

where $\theta$ is taken to satisfy $\theta^{2}=0$ and its trace is given as $\operatorname{tr} \theta=c$, where $c$ is a constant. Substituting (19) into $W(C)$, we have

$$
W(C)=\operatorname{Tr} P \exp \left\{\oint_{C}\left(\theta e_{\mu}^{a} J_{a}+\omega_{\mu}^{a} J_{a}\right) d x^{\mu}\right\}
$$

Within the path order operator, all the variables commute. So we can rewrite $W(C)$ as

$$
\begin{aligned}
& W(C)=\operatorname{Tr} P\left[\exp \oint_{C} \theta e_{\mu}^{a} J_{a} d x^{\mu} \exp \oint_{C} \omega_{\mu}^{a} J_{a} d x^{\mu}\right] \\
& =\operatorname{Tr} P\left[\sum_{n=0}^{\infty} \frac{\theta^{n}}{n !}\left(\oint_{C} e_{\mu}^{a} J_{a} d x^{\mu}\right)^{n} \exp \oint_{C} \omega_{\mu}^{a} J_{a} d x^{\mu}\right] .
\end{aligned}
$$


From the properties of $\theta$, we know that $\theta^{n}=0$ for any $n \geq 2$. Therefore

$$
\begin{aligned}
W(C) & =\operatorname{Tr} P \exp \left[\oint_{C} \omega^{a} J_{a}\right]+\operatorname{Tr} \theta P\left(\oint d x^{\mu} e_{\mu}^{a} J_{a} \exp \left[\oint_{C} \omega^{a} J_{a}\right]\right) \\
& =\operatorname{Tr} P \exp \left[\oint_{C} \omega^{a} J_{a}\right]+c \operatorname{Tr} \oint d x^{\mu} e_{\mu}^{a}(C(s)) U_{C}(s) \\
& =T^{0}[C]+c T^{1}[C] .
\end{aligned}
$$

We conclude that the covariant loop variables for spatial loops are determined by the Ashtekar $T$ variables.

If point particles are present, we locate them at punctures in space [11. We compute these two kinds of observables for loops $C$ which enclose only one puncture. In order to evaluate the observables, we first choose the representation of $I S O(2,1)$ given by

$$
\begin{gathered}
J_{0}=\frac{1}{2}\left(\begin{array}{cc}
i & 0 \\
0 & -i
\end{array}\right), J_{1}=\frac{1}{2}\left(\begin{array}{cc}
0 & i \\
-i & 0
\end{array}\right), J_{2}=\frac{1}{2}\left(\begin{array}{cc}
0 & 1 \\
1 & 0
\end{array}\right), \\
P_{a}=\theta J_{a} .
\end{gathered}
$$

In this representation we have [1]

$$
J_{a} J_{b}=-\frac{1}{4} \eta_{a b}+\frac{1}{2} \epsilon_{a b c} J^{c},
$$

Using the fundamental Poisson brackets and this representation of $S O(2,1)$, we compute the loop algebra for $T^{0}, T^{1}$ :

$$
\left\{T^{0}[\gamma], T^{0}[\delta]\right\}=0
$$

since the $T^{0}$ just depend on connection $\omega$, and

$$
\begin{aligned}
\left\{T^{1}[\gamma], T^{0}[\beta]\right\} & =\sum_{i} \Delta_{i}(\gamma, \beta)\left(T^{0}\left[\gamma \#_{i} \beta\right]-T^{0}\left[\gamma \#_{i} \beta^{-1}\right]\right) \\
\left\{T^{1}[\gamma], T^{1}[\beta]\right\} & =\sum_{i} \Delta_{i}(\gamma, \beta)\left(T^{1}\left[\gamma \#_{i} \beta\right]-T^{1}\left[\gamma \#_{i} \beta^{-1}\right]\right)
\end{aligned}
$$

where $i$ labels the points where the loops $\gamma$ and $\beta$ intesect, $\#_{i}$ stands for composition of two loops at the $i^{\text {th }}$ intersection. The structure constants of the loop algebra are

$$
\Delta_{i}(\alpha, \beta):=\int_{i} d s^{\mu} d t^{\nu} \delta^{2}(\alpha(s), \beta(t)) \epsilon_{\mu \nu}
$$


where the integrals are taken in an interval including the $i^{\text {th }}$ intersection. The number $\Delta_{i}$ is equal to minus or plus one depending on whether the dyad formed by the tangent vectors of the two loops at the $i^{\text {th }}$ intersection is left- or right-handed with respect to the orientation given by $\epsilon_{\mu \nu}$.

The particle associated with each puncture carries mass and angular momentum [11]. So at the puncture the constraints (12) are not enforced. Because of the constraints (12) we can make the loop $C$ infinitesimally close to the puncture in the covariant loop variable $W$. Using the infinitesimal condition, we can replace the path ordered line integral of the connection by the area integral of the curvature enclosed [11], i.e.,

$$
W(C)=\operatorname{Tr} \exp \left\{\theta j^{a} J_{a}+p^{a} J_{a}\right\},
$$

where

$$
p^{a}:=\int_{\delta \sigma} R^{a}, \quad j^{a}:=\int_{\delta \sigma} D e^{a},
$$

are the area integrals of the curvatures concentrated at the puncture. From the formula

$$
\exp (N \cdot J)=\cos (|N| / 2)+2 \sin (|N| / 2) \frac{N \cdot J}{|N|},
$$

and the tracelessness of $J_{a}$, we compute the covariant loop observables to be

$$
W(C)=\operatorname{Tr} \cos (|p+\theta j| / 2) .
$$

The normal of the vector is defined by

$$
|p+\theta j|:=(p+\theta j)^{a}(p+\theta j)_{a}=|p|+\theta p \cdot j /|p|
$$

where we have used the fact $\theta^{2}=0$. Again, applying this and expanding, we find that

$$
\cos [(|p|+\theta p \cdot j) / 2]=\cos (|p| / 2)-\theta p \cdot j \frac{\sin (|p| / 2)}{2|p|} .
$$

Finally the covariant loop observables for one puncture are:

$$
\begin{aligned}
W(C)= & \operatorname{Tr}\left[\cos (|p| / 2)-\theta p \cdot j \frac{\sin (|p| / 2)}{2|p|}\right] \\
& =2 \cos (|p| / 2)-c p \cdot j \frac{\sin (|p| / 2)}{|p|} .
\end{aligned}
$$


We get the same result by evaluating the $T$-variables and using the relation between the latter and covariant loops in (21).

We now briefly consider the quantum theory. We quantize in the reduced phase, as in Refs. [1, 8], for the special case of $\Sigma=T^{2}$, the flat 2-torus. We summarize the results here. Due to the nonconnectedness of the group $S O(2,1)$ and the Abelian property of the homotopy group of the 2-torus, the reduced phase space has disconnected sectors. For the time-like case, the reduced configuration space is topologically a 2 -torus. Let us choose $a, b$ as its coordinates, $a, b \in[0,1]$. The loop variable are promoted to operators $\hat{T}^{0}$ and $\hat{T}^{1}$ on the Hilbert space of $L^{2}$ functions $\Psi(a, b)$ over $\hat{\mathcal{C}}$ such that:

$$
\begin{array}{r}
\hat{T}^{0}[\alpha] \Psi(a, b)=2 \cos (\pi a) \Psi(a, b), \\
\hat{T}^{1}[\alpha] \Psi(a, b)=-2 \pi i \hbar \sin (\pi a) \frac{\partial}{\partial b} \Psi(a, b) .
\end{array}
$$

It is easy to check that the commutators of these operators are given by $-i \hbar$ times the value of the corresponding classical Poisson brackets. Hence the covariant loop variables on the 2-torus are given by the relation (21) as

$$
W(\alpha) \Psi(a, b)=\left\{2 \cos (\pi a)-2 c \pi i \hbar \sin (\pi a) \frac{\partial}{\partial b}\right\} \Psi(a, b) .
$$

\section{Observables for 4-D BF theory}

A careful analysis of Witten's approach to $2+1$ dimensional gravity reveals that the crucial reason for the solvability of the theory is that gravity in three dimensions has no local dynamics. The extension of this model to higher dimensions results in the BF theory with the following form of action [6, 7]:

$$
S=\operatorname{Tr} \int B \wedge F
$$

where $F$ is the curvature two-form of a connection $A$ on a principle bundle over the $n$-dimensional manifold $M$ with (simple) structure group $G . \quad B$ is a Lie algebra-valued $(n-2)$-form on $M$. On the 4 manifold $M=R \times \Sigma$ with structure group $G=S O(3,1)$, the action 
can be written as

$$
S=\int P_{a b} \wedge R^{a b},
$$

The action is put into the canonical form, with the result that the phase space variables are the $\omega_{i}^{a b}$ and their conjugate momenta $\pi_{a b}^{i}$, where

$$
\pi_{a b}^{i}=\epsilon^{i j k} P_{a b j k} .
$$

Here $a, b, \ldots$ are $\mathrm{SO}(3,1)$ indices and $i, j, \ldots$ are the spatial indices on $\Sigma$. The corresponding loop variables are, first, the $T^{0}$, given by

$$
T^{0}[\gamma]=\operatorname{Tr} U_{\gamma}(s),
$$

where

$$
U_{\gamma}(s)=P \exp \left(\oint_{\gamma} \omega^{a b} J_{a b}\right),
$$

with $\gamma$ a closed loop on $\Sigma$, and $J_{a b}$ the generators of $S O(3,1)$; and second the $T^{i}$, given by

$$
T^{i}[\gamma](s)=\operatorname{Tr}\left[\pi^{i}(\gamma(s)) U_{\gamma}(s)\right],
$$

which are linear in the momenta. Applying the fundamental Poisson bracket

$$
\left\{\omega_{i}^{a b}(x), \pi_{c d}^{j}(y)\right\}=\delta_{i}^{j} \delta_{c}^{[a} \delta_{d}^{b]} \delta(x, y),
$$

we can compute the loop algebra. Obviously, we have

$$
\left\{T^{0}[\gamma], T^{0}[\beta]\right\}=0 .
$$

The important Poisson bracket is between $T^{0}$ and $T^{i}$. First

$$
\begin{array}{r}
\left\{T^{0}[\gamma], T^{i}[\beta](t)\right\}=\left\{U_{\gamma}(s)_{I}^{I}, \pi^{i}(\beta(t))^{K}{ }_{L} U_{\beta}(t)^{L}{ }_{K}\right\} \\
=\int d x^{i} \delta(\beta(t), \gamma) U(s, t)^{I}{ }_{J} J_{a b}^{J M} U(t, s)_{M I} J^{a b K}{ }_{L} U_{\beta}(t)^{L}{ }_{K},
\end{array}
$$

where (41) has been used. If we choose the representation of generators $J_{a b}$ in $4 \times 4$ form 12 , we have

$$
J_{a b}{ }^{J}{ }_{M}=\delta_{a}^{J} \eta_{b M}-\delta_{b}^{J} \eta_{a M} .
$$

It follows that

$$
J_{a b}{ }^{J M} J^{a b K}{ }_{L}=2\left(\eta^{J K} \delta_{L}^{M}-\eta^{M K} \delta_{L}^{J}\right) .
$$


Using this result and noticing that $U_{\gamma}(s)_{I J}$ is an element of $S O(3,1)$, and satisfies the identity 円

$$
U_{\gamma}(s)_{I J}=U_{\gamma^{-1}}(s)_{J I},
$$

we obtain

$$
\left\{T^{0}[\gamma], T^{i}[\beta](t)\right\}=2 \int d x^{i} \delta^{3}(\beta(t), \gamma)\left[T^{0}(\gamma \# \beta)-T^{0}\left(\gamma \# \beta^{-1}\right)\right] .
$$

This expression contains distributional factors

$$
2 \int d x^{i} \delta^{3}(\beta(t), \gamma)
$$

as does the local expression(41), but with the difference that the distributional factors in (43) have support on curves rather than points. We may replace the $T^{i}$ by smeared loop variables in order to eliminate the distributional factors in the Poisson bracket. An elegant discussion is given by Smolin [13], and we merely recapitulate it here. Since the distributional factors in (43) are already one dimensional, the integral over the test functions should be two dimensional. It is natural to make this an integral over a surface. Hence we consider the dual of the momentum $\pi_{a b}^{i}$ on $\Sigma_{t}$, defined as

$$
\pi_{i j a b}^{*}=\frac{1}{2} \epsilon_{i j k} \pi_{a b}^{k}
$$

We note here that the $\pi_{i j a b}^{*}$ are the spatial projections of the components of the original variables $P_{a b}$. Correspondingly we have

$$
T_{i j}^{*}[\gamma](s)=\operatorname{Tr}\left[\pi_{i j}^{*}(\gamma(s)) U_{\gamma}(s)\right] .
$$

\footnotetext{
${ }^{1}$ We use the correspondence between Lorentz tensors and $\operatorname{SL}(2, \mathrm{C})$ spinors to write $U_{\gamma}(s)_{I J}$ as follows:

$$
U_{\gamma}(s)_{I J}=\sigma_{I}^{a a^{\prime}} \sigma_{J}^{b b^{\prime}} U_{\gamma}(s)_{a b} \bar{U}_{\gamma}(s)_{a^{\prime} b^{\prime}},
$$

where the matrices $U_{\gamma}(s)_{a b}$ and its conjugate are the "SL $(2, \mathrm{C})$ " representations of the holonomy operator along the curve $\gamma(s)$. It is shown in [1] that these satisfy:

$$
U_{\gamma}(s)_{a b}=-U_{\gamma^{-1}}(s)_{b a} ; \quad \bar{U}_{\gamma}(s)_{a^{\prime} b^{\prime}}=-\bar{U}_{\gamma^{-1}}(s)_{b^{\prime} a^{\prime}} .
$$
}

Hence

$$
U_{\gamma}(s)_{I J}=\sigma_{I}^{a a^{\prime}} \sigma_{J}^{b b^{\prime}} U_{\gamma^{-1}}(s)_{b a} \bar{U}_{\gamma^{-1}}(s)_{b^{\prime} a^{\prime}}=U_{\gamma^{-1}}(s)_{J I} .
$$


Let us consider a one-parameter continuous family of loops $\gamma^{i}(s, u)$ where $u \in[0,1]$ such that they form a strip, which we denote with a hat $\hat{\gamma}$. For each $u, \gamma^{i}(s)_{u} \equiv \hat{\gamma}^{i}(s, u)$ is a closed loop. The parameters $s$ and $u$ then coordinatize the two dimensional surface of the strip. The following smeared version of the $T^{1}$ observable can be defined $\mathbb{F}^{2}$ :

$$
T^{1}[\hat{\gamma}]:=\frac{1}{\pi^{2}} \int d u \int d s \frac{\partial \hat{\gamma^{i}}}{\partial u} \frac{\partial \hat{\gamma}^{j}}{\partial s} T_{i j}^{*}[\gamma](s)
$$

The Poisson bracket of this observable with $T^{0}[\alpha]$ is expressed in terms of the intersection number of loop $\alpha$ and the strip $\hat{\gamma}$. If the loop does not intersect the strip, the Poisson bracket is zero. If the loop intersects the strip, at intersection $n$, we have

$$
I_{n}(\hat{\gamma}, \alpha)=\int_{\sigma_{n}} d S^{i j} \int d x^{k} \epsilon_{i j k} \delta^{3}(\hat{\gamma}, \alpha),
$$

which is equal to \pm 1 according to the orientation of the intersection. In the above $\sigma_{n}$ denotes the neighbourhood of the intersection $n$. Finally, the loop algebra becomes

$$
\begin{aligned}
\left\{T^{0}[\alpha], T^{1}[\hat{\gamma}](t)\right\} & =\sum_{n} I_{n}(\hat{\gamma}, \alpha)\left(T^{0}\left[\alpha \#_{n} \hat{\gamma}\right]-\left(T^{0}\left[\alpha \#_{n} \hat{\gamma}^{-1}\right]\right),\right. \\
\left\{T^{1}[\hat{\alpha}], T^{1}[\hat{\gamma}](t)\right\} & =\sum_{n} I_{n}(\hat{\gamma}, \hat{\alpha})\left(T^{1}\left[\hat{\alpha} \#_{n} \hat{\gamma}\right]-\left(T^{1}\left[\hat{\alpha} \#_{n} \hat{\gamma}^{-1}\right]\right) .\right.
\end{aligned}
$$

The geometrical significance of the loop algebra is that it depends on the intersection number of loops or strips with other loops or strips.

We know that $T$-variables are not the physical observables in $3+1$ Einstein gravity since they are not diffeomorphism invariant. However, the model that we consider here, unlike Einstein gravity, is a topological field theory. For the BF theory considered here, it turns out that $T^{0}$ and $T^{1}$ are physical observables, i.e. they commute with all the constraints. The constraints of the 4-D BF theory are

$$
\begin{aligned}
R^{a b} & \approx 0, \\
C_{a b}:=D_{i} \pi_{a b}^{i} & \approx 0 .
\end{aligned}
$$

\footnotetext{
${ }^{2}$ The normalization here differs by the factor $\pi^{-2}$ from the literature, e.g. Ref.[8]. It is necessary to choose this normalization in order to get the expression (69) in the form given in the literature without clumsy factors of $\pi$.
} 
Note that the constraints $C_{a b}$ satisfy

$$
\left\{C_{a b}(x), C_{c d}(y)\right\}=\frac{1}{2}\left[\eta_{c b} C_{a d}+\eta_{b d} C_{c a}+\eta_{a c} C_{d b}+\eta_{a d} C_{b c}\right] \delta(x, y) .
$$

This is the algebra of $S O(3,1)$, so the $C_{a b}$ are the generators of the gauge group $S O(3,1)$. The loop variables $T^{0}$ and $T^{1}$ are gauge invariant. Thus it follows naturally that

$$
\begin{gathered}
\left\{T^{0}[\gamma], D_{i} \pi_{a b}^{i}\right\}=0 \\
\left\{T^{1}[\hat{\gamma}], D_{i} \pi_{a b}^{i}\right\}=0
\end{gathered}
$$

Since the $T^{0}$ are just functions of the configuration variables, as are $R^{a b}$, it follows trivially that

$$
\left\{T^{0}[\gamma], R^{a b}\right\}=0
$$

The crucial Poisson bracket is that between $T^{1}$ and $R^{a b}$. Analogous to the $2+1$ dimensional theory, we have

$$
\left\{T^{1}[\hat{\gamma}], R^{a b}\right\}=\frac{1}{\pi^{2}} \int_{\sigma} D\left[D\left(\delta_{c d}^{a b} \delta^{3}(\gamma(s), x) \operatorname{Tr} J^{c d} U_{\gamma}(s)\right)\right] \sim R^{a b},
$$

where $\sigma$ is the volume enclosed by the strip $\hat{\gamma}$ in $\Sigma$, and we have used Stokes theorem [14]. This bracket weakly vanishes by means of the constraint. This is the desired result.

For this topological model, the reduced phase space is also a moduli space of flat connections modulo $\mathrm{SO}(3,1)$ gauge invariance, as in $2+1$ gravity. It is interesting to consider the observables on a topologically nontrivial underlying spatial 3-manifold $\Sigma$ which has the topology of a 3-torus $T^{3}$ 15. First it is useful to discuss some features of the holonomy. If, as we assume, reduced phase space can be polarized, then it is the tangent bundle over the reduced configuration space $\hat{\mathcal{C}}$. Each element $\omega$ of $\hat{\mathcal{C}}$ is determined by fixing a base point $p$ on $\Sigma$ and specifying the holonomies, modulo the action of $S O(3,1)$ at $p$, of $\omega$ around the $n$ generators of the homotopy group of $\Sigma$. The holonomies provide us $n$ gauge group elements, $\left(U_{1}, \ldots U_{n}\right)$, and $\omega$ is determined by the equivalence class $U \cdot\left(U_{1}, \ldots U_{n}\right) \cdot U^{-1}$ where $U$ is the gauge group action at $p$. $\hat{\mathcal{C}}$ has several disconnected components 
essentially because the Lie algebra $S O(3,1)$ has three disjoint orbits under the natural action of the group. Each $U_{k}$ is a rotation either along a time-like, null, or space-like axis and the action of the gauge group at $p$ must map that $U_{k}$ to a rotation with the same type of axis. For the case that $\Sigma=T^{3}$, the flat 3 -torus, we have three generators for the homotopy group which are denoted as $\alpha_{1}, \alpha_{2}, \alpha_{3}$. Now, since the homotopy group of $T^{3}$ is Abelian, it follows that the holonomy group of any flat connection must also be Abelian. A general element of the homotopy group of the torus may be written as

$$
\alpha=\alpha_{1}^{n_{1}} \alpha_{2}^{n_{2}} \alpha_{3}^{n_{3}}
$$

where $n_{1}, n_{2}, n_{3}$ are integers which represent the number of times the loop winds the three generators. The holonomies satisfy

$$
\left[U_{\alpha_{1}}, U_{\alpha_{2}}\right]=\left[U_{\alpha_{3}} U_{\alpha_{2}}\right]=\left[U_{\alpha_{1}}, U_{\alpha_{3}}\right]=0 .
$$

Therefore they are $S O(3,1)$ rotations around the same axis. Under gauge transformations the axis itself rotates preserving only its timelike, null, or space-like character. This immediately divides $\hat{\mathcal{C}}$ into three sectors. In the time-like case, the subgroup of $S O(3,1)$ is the group of 3-dimensional rotations $S O(3)$ [12] which is compact. Thus this sector has the topology $T^{3}$. In the null sector, the corresponding subgroup is the Euclidian group in 2-dimensions, $E_{2}$. Finally in the space-like case, the subgroup of $S O(3,1)$ is the 3-dimensional Lorentz group $S O(2,1)$. In the cases of null and spacelike sectors, the topologies are more complicated. By equation (58), we should consider the Abelian subgroup in those sectors. Since the groups $S O(2,1)$ and $E_{2}$ contain different Abelian subgroups, they may correspond to different topologies.

We now discuss the reduced phase quantization for the time-like case in detail. We parameterize an arbitrary flat connection as follows:

$$
\omega=\left(\sum_{j=1}^{3} a_{j} d \theta_{j}\right) \frac{i \tau^{3}}{2},
$$

for some choice of the real constant $a_{j}$, where $\theta_{j}$ are the three angular coordinates on $\Sigma$ and $\frac{i \tau^{3}}{2}$ one of generators of $S O(3)$ with

$$
\tau^{3}=\left(\begin{array}{cc}
1 & 0 \\
0 & -1
\end{array}\right) .
$$


Let us choose the loops to wind once around the generator $\alpha_{1}$. As in the $2+1$ dimensional case, we promote the loop variables to operators on the Hilbert space of $L^{2}$ functions $\Psi\left(a_{1}, a_{2}, a_{3}\right)$ over the reduced configuration space. It then follows that:

$$
\begin{array}{r}
\hat{T}^{0}\left[\alpha_{1}\right] \psi\left(a_{1}, a_{2}, a_{3}\right)=2 \cos \left(a_{1} \pi\right) \psi\left(a_{1}, a_{2}, a_{3}\right), \\
\hat{T}^{1}\left[\hat{\alpha}_{1}\right] \Psi\left(a_{1}, a_{2}, a_{3}\right)=\frac{1}{\pi^{2}} \int d u^{i} \int d s^{j} \frac{1}{2} \epsilon_{i j k} \operatorname{Tr}\left[\pi^{k} U_{\alpha_{1}}(s)\right] \Psi\left(a_{1}, a_{2}, a_{3}\right) .
\end{array}
$$

Since on $\alpha_{1}$ the loop has just one component, i.e. $d s^{j}=d \theta^{1}$, we get

$$
\begin{array}{r}
\hat{T}^{1}\left[\hat{\alpha_{1}}\right] \Psi\left(a_{1}, a_{2}, a_{3}\right)= \\
\int_{0}^{1} d u \int_{0}^{2 \pi} d \theta^{1} \frac{1}{2}\left\{\operatorname{Tr}\left[\pi^{2} U_{\alpha_{1}}(s)\right]-\operatorname{Tr}\left[\pi^{3} U_{\alpha_{1}}(s)\right]\right\} \Psi\left(a_{1}, a_{2}, a_{3}\right) \\
=-\frac{1}{\pi} i \hbar \sin \left(\pi a_{1}\right)\left[\frac{\partial}{\partial a_{2}}-\frac{\partial}{\partial a_{3}}\right] \Psi\left(a_{1}, a_{2}, a_{3},\right) .
\end{array}
$$

Generally, we thus have

$$
\begin{array}{lc}
\hat{T}^{0}\left[\alpha_{i}\right] \Psi\left(a_{1}, a_{2}, a_{3}\right)= & 2 \cos \left(a_{i} \pi\right) \Psi\left(a_{1}, a_{2}, a_{3}\right), \\
\hat{T}^{1}\left[\hat{\alpha}_{i}\right] \Psi\left(a_{1}, a_{2}, a_{3}\right)= & -\frac{1}{\pi} i \hbar \sin \left(\pi a_{i}\right) \sum_{j k} \epsilon_{i j k} \frac{\partial}{\partial a_{j}} \Psi\left(a_{1}, a_{2}, a_{3},\right) .
\end{array}
$$

Since $\hat{T}^{1}$ is linear in the momenta, it can always be written as

$$
\hat{T}^{1}=\sum v^{a}(q) p_{a}
$$

at the point $(q, p)$ in the reduced phase space, where $v^{\alpha}(q)$ is a vector field on the configuration space. It is then the vector field associated with the operator $\hat{T}^{1}$ by:

$$
\hat{T}^{1}[\hat{\alpha}] \Psi(\omega)=\frac{\hbar}{i} \mathcal{L}_{v_{\hat{\alpha}}} \Psi(\omega),
$$

where $\mathcal{L}$ is the Lie derivative on $\mathcal{C}$. In the case of $\Sigma=T^{3}$,

$$
v_{\hat{\alpha}}^{i}=\int d s d t \epsilon^{i j k} \delta^{3}(x, \hat{\alpha}(s, t)) \partial_{s} \alpha_{j} \partial_{t} \alpha_{k} \operatorname{Tr}\left(\tau^{3} U_{\hat{\alpha}}\right) .
$$

We now quantize in the loop representation. The quantum theory is given in terms of a representation of the loop algebra as an operator 
algebra [1, 8]. The usual canonical quantization consists of a representation of the canonical Poisson algebra. By the gauge property of the theory, we can construct a representation on the gauge constraint $C_{a b}=0$ surface of the phase space. The representation space $\mathcal{S}$ will consist of functionals of loops in $\Sigma, \mathcal{A}[\alpha] \in \mathcal{S}$. The action of $\hat{T}^{0}[\alpha]$ and $\hat{T}^{1}[\hat{\alpha}]$ can be expressed in this representation as follows:

$$
\begin{gathered}
\left(\hat{T}^{0}[\alpha] \mathcal{A}\right)[\gamma]:=\mathcal{A}[\alpha \# \gamma]+\mathcal{A}\left[\alpha \# \gamma^{-1}\right] \\
\left(\hat{T}^{1}[\hat{\alpha}] \mathcal{A}\right)[\{\gamma\}]:=i \hbar \sum_{n} I_{n}(\hat{\alpha}, \gamma)\left(\mathcal{A}\left[\hat{\alpha} \#{ }_{n} \gamma\right]-\mathcal{A}\left[\hat{\alpha} \#_{n} \gamma^{-1}\right]\right),
\end{gathered}
$$

where we can use any loop $\gamma$ in the same homotopy class; the result is independent of the choice. This representation satisfies the following properties:

$$
\begin{array}{ll} 
& \hat{T}^{0}[\alpha]=\hat{T}^{0}\left[\alpha^{-1}\right], \quad \hat{T}^{1}[\hat{\alpha}]=\hat{T}^{1}\left[\hat{\alpha}^{-1}\right], \\
& \hat{T}^{0}[\alpha \# \gamma]=\hat{T}^{0}[\gamma \# \alpha], \quad \hat{T}^{1}[\hat{\alpha} \# \hat{\gamma}]=\hat{T}^{1}[\hat{\gamma} \# \hat{\alpha}] ; \\
\text { (2) } & \hat{T}^{0}[0]=d, \hat{T}^{1}[0]=0 \\
(3) & \hat{T}^{0}[\alpha \# \gamma]+\hat{T}^{0}\left[\alpha \# \gamma^{-1}\right]=\hat{T}^{0}[\alpha] \hat{T}^{0}[\gamma] \\
\text { (4) } & {\left[\hat{T}^{A}, \hat{T}^{B}\right]=i \hbar\left\{T^{A}, T^{B}\right\}, \quad A, B=0,1 ;} \\
\text { (5) } & \hat{T}^{0}[\alpha]=\left(\hat{T}^{0}[\alpha]\right)^{*} \text { is real. }
\end{array}
$$

In (2) $d$ is the dimensions of the group representation.

In a given connected sector of the reduced phase space $\hat{\mathcal{C}}$, denoted by $\hat{\mathcal{C}}_{i}$, the transformation between the two sorts of representations is [16]:

$$
\mathcal{A}[\alpha]=\int_{\hat{\mathcal{C}}_{i}} d V\left(T^{0}[\alpha](\omega)\right) \Psi(\omega),
$$

where $d V$ is a volume element on $\hat{\mathcal{C}}_{i}$ and $\Psi(\omega)$ is the wave function in the connection representation.

Returning to the case of the three torus discussed above, by choosing the volume element $d V=d a_{1} d a_{2} d a_{3}$ on the torus, we could construct the transform explicitely in the time-like sector:

$$
\mathcal{A}\left[n_{1}, n_{2}, n_{3}\right]=\int_{0}^{1} d a_{1} \int_{0}^{1} d a_{2} \int_{0}^{1} d a_{3} \cos \left(\pi n_{i} a_{i}\right) \Psi\left(a_{1}, a_{2}, a_{3}\right),
$$

where $n_{i} a_{i}=n_{1} a_{1}+n_{2} a_{2}+n_{3} a_{3}$. 
The fundamental operators $\hat{T}^{0}, \hat{T}^{1}$ in the loop representation are

$$
\begin{array}{r}
\hat{T}^{0}\left[\alpha_{1}\right] \mathcal{A}\left[n_{1}, n_{2}, n_{3}\right]=\mathcal{A}\left[n_{1}+1, n_{2}, n_{3}\right]+\mathcal{A}\left[n_{1}-1, n_{2}, n_{3}\right] \\
\hat{T}^{1}\left[\hat{\alpha}_{1}\right] \mathcal{A}\left[n_{1}, n_{2}, n_{3}\right]= \\
i \hbar\left(n_{2}-n_{3}\right)\left(\mathcal{A}\left[n_{1}+1, n_{2}, n_{3}\right]-\mathcal{A}\left[n_{1}-1, n_{2}, n_{3}\right]\right)
\end{array}
$$

and similarly for the operators associated with the other two homotopy group generators $\alpha_{2}, \alpha_{3}$. Noticing that the transform (67) is just a cosine Fourier transform, we can represent the wave functions in the connection representation in terms of the wave functions in the loop representation. The inverse transform is

$$
\Psi\left(a_{1}, a_{2}, a_{3}\right)=\mathcal{A}[0,0,0]+2 \sum_{n_{1} n_{2} n_{3}} \mathcal{A}\left[n_{1}, n_{2}, n_{3}\right] \cos \left(\pi n_{i} a_{i}\right) .
$$

This is a demonstration that the representation of the loop algebra is (over) complete. Any wave function can be expanded as a linear combination of the states $\mathcal{A}\left[n_{1}, n_{2}, n_{3}\right]$.

\section{Discussion}

Comparing the 4-D BF theory and Einstein gravity, we see that the constraint structure of these two theories are quite different. In terms of Ashtekar's variables, the constraints of Einstein gravity are [1]:

$$
\begin{aligned}
& \mathcal{G}_{a}=\mathcal{D}_{i} \tilde{E}_{a}^{i} \approx 0, \\
& \mathcal{V}_{i}=\tilde{E}_{a}^{j} F_{i j}^{a} \approx 0, \\
& \mathcal{S}=\epsilon^{a b c} F_{i j a} \tilde{E}_{b}^{i} \tilde{E}_{c}^{j} \approx 0 .
\end{aligned}
$$

They are usually called, respectively, the Gauss constraint, the vector constraint and the scalar (or Hamiltonian) constraint. The loop variables are weakly annihilated by the Gauss and the scalar constraints, but not by the vector constraint since the latter generates diffeomorphisms. In contrast, in the 4-D BF theory, the vector constraint and the scalar constraint are equivalent to the constraint that the $S O(3,1)$ connection is flat. Thus there are only two constraints,

$$
R_{i j}^{a b} \approx 0, \quad C_{a b} \approx 0,
$$


in the BF theory. They generate the spatial projections of the gauge transformations. The gauge invariant loop variables naturally commute with these constraints. Furthermore, in the BF theory diffeomorphisms are generated by the constraints [6]. In this sence, the loop variables are the physical observables.

Finally, let us note an interesting difference between the loop representation of the $T$-variables on $T^{3}$ in the 4 -D BF theory and the loop representation of the $T$-variables on $T^{2}$ in the 3 - $\mathrm{D}$ gravity theory [8, 16]. Equations (68) and (70) tell us that on $T^{3}$, not only is the trivial state $\mathcal{A}[0,0,0]$ annihilated by $\hat{T}^{1}$, but so are all the states $\mathcal{A}[n, n, n]$ for any integer $n$. Furthermore, these are the only states which are annihilated by $\hat{T}^{1}$. Hence, in the connection representation, the state $\Psi_{0}(\omega)$ with $\hat{T}^{1} \Psi_{0}(\omega)=0$ can be written as

$$
\Psi_{0}(\omega)=\sum_{n} c_{n}(\omega) \mathcal{A}[n, n, n]
$$

where $n$ are arbitary integers.

We can also construct these observables in another simple topological space, namely $\Sigma=S^{2} \times S^{1}$. Since $\Sigma$ is now a product space, the fundamental group is a product 17]:

$$
\pi_{1}(\Sigma)=\pi_{1}\left(S^{2}\right) \times \pi_{1}\left(S^{1}\right) .
$$

But since $\pi_{1}\left(S^{2}\right)=\{e\}$,

$$
\pi_{1}(\Sigma)=\pi_{1}\left(S^{1}\right)
$$

The homotopy group of $\Sigma$ has only one generator, denoted $\alpha$. Hence the homotopy of any single loop $\Gamma$ is labeled by the integer $n$, such that $\Gamma=\alpha^{n}$. The non-trivial holonomies are generated by a single homotopy class, and hence are determined by a single axis of given causality. Let $\left(\theta, \theta^{\prime}, \phi\right)$ be the coordinates on $\Sigma$, such that $\left(\theta, \theta^{\prime}\right)$ are the coordinates of $S^{2}$. The ranges of the variables are then $0 \leq \theta, \phi<$ $2 \pi, 0 \leq \theta^{\prime}<\pi$. The configuration space $\hat{\mathcal{C}}$ is one dimensional. For the time-like axis, the abelian subgroup of $S O(3,1)$ is $S O(2)$, and hence the topology of this sector is $S^{1}$. Introducing the coordinate $a \in[0,1]$ on it, we may choose a flat connection on $\Sigma$ representing that point of $\hat{\mathcal{C}}$ :

$$
\omega^{b}=\omega_{i}^{b} d x^{i}=a\left(\partial_{i} \phi\right) d x^{i} \tau^{b}=a d \phi \tau^{b}
$$


where $\tau^{b}$ is the generator of $S O(2)$. If we choose the dual of the momenta as

$$
\pi_{i j}^{* a}=p \partial_{i} \theta^{A} \partial_{j} \phi \tau^{b},
$$

with $n$ denoting the winding number of the loop, then the $T$ observables are given by

$$
\hat{T}^{0}[n](a)=2 \cos (n \pi a) \quad, \quad \hat{T}^{1}[n](a, p)=2 \pi p \sin (n \pi a) .
$$

\section{Acknowledgments}

The authors wish to acknowledge the partial support of the Natural Sciences and Engineering Research Council of Canada.

\section{References}

[1] A. Ashtekar. Non-Perturbative Canonical Gravity World Scientific, Singapore, 1990.

[2] T. Jacobson, L. Smolin. Nucl. Phys. B299 (1988)295

[3] C. Rovelli, L. Smolin. Nucl. Phys. B331 (1990)80.

[4] J. Pullin, "Knot Theory and Quantum Gravity in Loop Space: A Primer", Univ. of Utah Preprint UU-REL-93/1/9, 1992.

[5] A. Achucarro, P. Townsend Phys. Lett. $180 B$ (1986) 85; E. Witten. Nul. Phys. B311 (1989)46.

[6] G. T. Horowitz. Commun. Math. Phys. 125 (1989)417.

[7] M. Blau and G. Thompson. Phys. Lett. B228 (1989)64.

[8] L. Smolin. Loop representation for quantum gravity in $2+1$ dimensions.Proc. 12th Johns Hopkins workshop: Topology and Quantum Field Theory(Florence, Italy) (1990) (Singapore: World Scientific). A. Ashtekar, V. Husain, C. Rovelli, J. Samuel, L. Smolin. Class. Quantum Grav. 6 (1989)185. 
[9] H. Y. Lee, A. Nakamichi and T. Ueno, Phys. Rev. D47, (1993) 1563; A. Nakamichi, "Wave Function of the UNiverse in Topological and in Einstein 2-form Gravity", Tokyo Inst. of Tech. Preprint 214/COSMO28, 1993.

[10] C-B. Kim, T. Shimizu, K. Yoshida. Class. Quantum Grav. 9 (1992)1211.

[11] S.P. Martin. Nul. Phys. B327 (1989)178.

[12] W-K. Tung. Group Theory in Physics World Scientific, Philadephia, Singapore. 1985.

[13] L. Smolin. Recent Developments in Nonperturbative Quantum Gravity, Preprint 1992.

[14] N.E. Bralic. Phys. Rev. D 22 (1980)3090; M.B. Mensky. Lett. Math. Phys. 3 (1979)513.

[15] A. Ashtekar, C.J. Isham. Class. Quantum. Grav. 9 (1992)1433.

[16] A. Ashtekar, V. Husain, C. Rovelli, J. Samuel, L. Smolin. Class. Quantum. Grav. 6 (1989)L185.

[17] S-T Hu. Homotopy Theory Academic Press, New York and London, 1959. 